\title{
IMT SIM: beyond the realms of resuscitation and procedures
}

\author{
Authors: Christopher Huntley, ${ }^{*}$ Joseph Wheeler, Jennifer Kerks, Stuart Hamilton, Amanda Szuszman, Philip Dainty \\ and Richard Barlow
}

\section{Introduction}

The impending arrival of the new internal medicine training (IMT) curriculum has shifted focus from knowledge-based assessments towards capabilities in practice (CiP) and generic capabilities in practice (GCP). These focus on non-technical professional skills and medical leadership, providing an opportunity for Simulation (SIM) Based Education (SBE) to support IMT development and experience. The benefits of SBE are far beyond traditional skills and drills training (such as resuscitation and procedures), as previously touted. We present our new IMT SIM training programme at the end of its pilot year.

\section{Method}

The IMT SIM programme is delivered at SimWard-Wolverhampton and provides half-day simulation-ward training to a maximum of five core medical training (CMT) or IMT doctors per session. The programme content maps to the new IMT curriculum, layering common medical inpatient and outpatient scenarios with varying levels of complex non-technical human factor themes and professional and leadership skills. All scenarios are adapted from events that faculty members have encountered through their physician training.

Themes encompassed throughout the programme include, but are not limited to: situational awareness; managing and resolving conflict; prioritisation; ethical issues in practice; team working; clinical reasoning; bias in practice; leadership; and followship. Feedback from candidates was collated in each session through Likert scales and unstructured qualitative questions to refine future scenarios.

\section{Results and discussion}

Seventeen CMT doctors undertook the pilot IMT SIM programme between June 2018 and February 2019, with nine returning surveys. $100 \%$ felt the sessions were 'very relevant' to their clinical practice and $100 \%$ felt they learnt 'a great deal' from the sessions. $89 \%$ felt the aims of the sessions were 'very clear' and $66 \%$ felt that the intended learning outcomes were 'very clear'. $89 \%$ felt that

Authors: New Cross Hospital, Wolverhampton ${ }^{*} \mathrm{RCP}$ chief registrar their practice would change either 'a great deal' or 'quite a lot' as a result of the sessions.

From unstructured responses, $66 \%$ of candidates found the nontechnical and human factor elements of each scenario extremely beneficial. Other feedback highlighted a safe environment enhancing the learning in the debrief, the diversity of scenarios and the differing complexities and themes arising. $44 \%$ of candidates specifically requested further sessions within their training. Faculty members observed that despite each session including the same scenarios, differing themes emerged during the debrief as a result of the varying past experiences of candidates. This allowed the tailoring of sessions to each candidate.

\section{Conclusion}

The pilot year of IMT SIM has highlighted the benefit of SBE in providing training in non-technical aspects of clinical practice, and should not only be used in resuscitation and procedural training. SBE is still under-utilised and this fantastic educational resource should complement other forms of medical education. As a result of the success of this pilot year, our faculty is now expanding IMT SIM, with separate IMT year 1, 2 and 3 sessions in development. Our long-term vision is to provide this programme regionally. 